Check for updates

Cite this: J. Anal. At. Spectrom., 2020, 35,1482

\title{
Comment on: "Slope ratio calibration for analysis of plant leaves by laser-induced breakdown spectroscopy" by Lidiane C. Nunes, Fabio R. P. Rocha and Francisco J. Krug, JAAS, 2019, 34, 2314
}

\author{
Vincenzo Palleschi (iD
}

Received 11th November 2019

Accepted 15th May 2020

In the paper "Slope ratio calibration for analysis of plant leaves by laser-induced breakdown spectroscopy", recently published on JAAS, the authors present an allegedly new calibration method for LIBS analysis, called Slope Ratio Calibration (SRC). In this short comment we will demonstrate that this method is completely equivalent to Single-Point Calibration (SPC), when a fair comparison between the two methods is done.

rsc.li/jaas

\section{Introduction}

In ref. 1, Numes et al. presented a method for quantitative analysis of LIBS spectra based on the building of a calibration curve using a single reference standard. The slope $b_{\text {std }}$ of this calibration curve is then compared with the one obtained applying the same procedure to the unknown sample $\left(b_{\text {sample }}\right)$, obtaining the concentration of the analyte in the unknown sample as:

$$
C_{\text {sample }}=C_{\text {std }} \frac{b_{\text {sample }}}{b_{\text {std }}}
$$

Even without any knowledge of the LIBS technique, the possibility of obtaining a meaningful calibration curve using only a single reference sample, without diluting it or adding external standards, seems to be logically impossible.

In fact, the solution proposed by the authors of ref. 1 is to obtain a calibration curve plotting the analyte LIBS line intensity not as a function of the concentration of the analyte, as usual, but as a function of the number of accumulations of the LIBS signal on the detector. The same procedure is repeated for the unknown sample, and the slopes of the resulting curves are then used in eqn (1).

It's evident that this method, at the best, could be used for checking the linearity of the detector used, but would not give any information about the chemical or physical mechanism that could produce a non-linear response of the system (for example, matrix effects and/or self-absorption in the LIBS plasma).

Applied and Laser Spectroscopy Laboratory, Research Area of CNR, Via G. Moruzzi, 1, 56124 Pisa, Italy. E-mail: vincenzo.palleschi@cnr.it
The signal obtained accumulating on the detector $n$ spectra from a sample with a given concentration $C$ of the analyte in NOT equivalent to the one that would have been obtained from a sample with concentration $n \times C$, unless all the processes in the plasma are linear. In this latter case there is no need for building a 'calibration curve', and definitely not in the way suggested by the authors.

A simple calculation would support the logic of the previous reasoning. Let's assume the 'calibration curve' is built using only two points, one corresponding to $n_{1}$ accumulations and the other to $n_{2}>n_{1}$ accumulations. If the detector is working properly, giving a signal which is proportional to the intensity of the line chosen for the calibration, the signal corresponding to $n_{1}$ accumulations would be $n_{1} \times I$, and the one corresponding to $n_{2}$ accumulations would be $n_{2} \times I$, where $I$ is the intensity of the line corresponding to a single accumulation. Consequently, the slope of the 'calibration curve' on the standard would be:

$$
b_{\mathrm{std}}=\frac{n_{2} I_{\mathrm{std}}-n_{1} I_{\mathrm{std}}}{n_{2}-n_{1}}=I_{\mathrm{std}}
$$

In the same way, the one calculated on the sample would be:

$$
b_{\text {sample }}=\frac{n_{2} I_{\text {sample }}-n_{1} I_{\text {sample }}}{n_{2}-n_{1}}=I_{\text {sample }}
$$

which makes eqn (1) completely equivalent to the Single Point Calibration expression:

$$
C_{\text {sample }}=C_{\text {std }} \frac{I_{\text {sample }}}{I_{\text {std }}}
$$

In ref. 1, Nunes et al. made an unfair comparison between the results of their 'new' method and the classical Single-Point Calibration results, averaging the line intensities only over a maximum of 30 spectra for SPC, while for SRC $30+20+15+10$ 
$+5=80$ spectra where used (see Fig. 5 in the original manuscript). This explains the apparently better results obtained by SRC with respect to SPC.

\section{Conclusion}

We have shown that the SRC method proposed by Nunes et al. in ref. 1 is completely equivalent to Single-Point Calibration. As SPC, the SRC method assumes a linear dependence of the emitted light intensity on the analyte concentration and, as SPC, it cannot give information about matrix or self-absorption effects which often affect the precision of LIBS analysis. Finally, we would take the occasion for stressing once again that doing quantitative analysis by LIBS is not trivial, and 'mathemagical' (in this case, really, 'arithmagical') approaches do not help, without a deep comprehension of the chemical and physical processes underlying the analysis.

\section{Conflicts of interest}

There are no conflicts to declare.

\section{References}

1 L. C. Nunes, F. R. P. Rocha and F. J. Krug, Slope ratio calibration for analysis of plant leaves by laser-induced breakdown spectroscopy, J. Anal. At. Spectrom., 2019, 34, 2314, DOI: 10.1039/c9ja00270g. 